\title{
Aplicación del Modelo de Gestión Pirámide del Desarrollo Universitario en la Universidad de Otavalo, Ecuador
}

\author{
Antonio de J. Romillo y Cecilia J. Polaino \\ Universidad de Otavalo, Cdla Imbaya, Ave. de los Sarances $s / n$ y Pendoneros, Cantón Otavalo, Provincia \\ Imbabura, Ecuador. (e-mail: aromillo@uotavalo.edu.ec; cpolaino@uotavalo.edu.ec.)
}

Recibido Jun. 15, 2018; Aceptado Ago. 8, 2018; Versión final Ago. 30, 2018, Publicado Feb. 2019

\begin{abstract}
Resumen
El objetivo de este trabajo es determinar la contribución de la aplicación del Modelo de Gestión Pirámide del Desarrollo Universitario en los resultados de la Universidad de Otavalo. El modelo integra las áreas de resultados clave, las restricciones de desarrollo, el contexto institucional y la dirección estratégica de la organización, y fue el resultado de una investigación previamente realizada en la dirección estratégica de cuatro instituciones de educación superior. Se conceptualiza el modelo y se explica la metodología, propia de una investigación correlacional con diseño cuasi experimental, mediante la cual se aplicó la Pirámide del Desarrollo Universitario en la institución. Los resultados obtenidos en tres años muestran mejoras en los indicadores de calidad de claustro, formación académica, investigación, vinculación con la sociedad y aseguramiento logístico posibilitando la acreditación de la universidad en el año 2016. Se concluye que la aplicación del modelo ha significado una importante contribución a la gestión institucional.
\end{abstract}

Palabras clave: modelo de gestión; educación superior; dirección estratégica; desarrollo universitario; acreditación

\section{Application of the University Development Pyramid Management Model at the University of Otavalo, Ecuador}

\begin{abstract}
The objective of this paper is to determine the contribution of the application of the University Development Pyramid Management Model in the results of the University of Otavalo. The model integrates the key results areas, the development constraints, the institutional context and the strategic direction of the organization, and was the result of a research previously carried out in the strategic direction of four higher education institutions. The model is conceptualized and the methodology is explained, typical of a research of the quasi-experimental correlational type, by means of which the Pyramid of University Development was applied in the institution. The results obtained during three years show improvements in the quality indicators of academic training, research, link with society, and logistics assurance, enabling the accreditation of the university in 2016. It is concluded that the application of the model represents an important contribution to the institutional management.
\end{abstract}

Keywords: management model; higher education; strategic management; university development; accreditation 


\section{INTRODUCCIÓN}

Numerosos y diversos son los modelos de gestión universitaria, muchos se enfocan hacia los procesos sustantivos de la universidad: modelos pedagógicos de gestión docente o académica, educativos centrados en el estudiante, en competencias, didácticos u otros (Abreu, Naranjo, Rhea y Gallegos, 2016; Acosta, Abreu y Coronel, 2015; Mintz, 2014); de vinculación con la sociedad o extensión universitaria (Polaino y Romillo, 2017; Loi y Di Guardo, 2015; Dan, 2012; Montesinos, Carot, Martínez y Mora, 2008); de investigación o innovación (Larrea, 2014; Henningsen, Schlaeger y Tenorth, 2013). En cada uno de estos modelos podemos encontrar a su vez diferentes enfoques, así por ejemplo entre los modelos de investigación e innovación están: los modelos lineales de empujón de la ciencia y de jalón del mercado (Nowotny, Scott, y Gibbons, 2003); modelos interactivos (Osei-Bryson, Mansingh y Rao, 2014; Patel y Gorvadiya, 2014); el Modelo de la Triple Hélice (Etzkowitz, 2014) y otros.

Existen además modelos de gestión universitaria que centran su atención en el tipo de gobernabilidad de la institución de la educación superior. Lolas (2006) los clasifica en: autoritarios, participativos, empresarial, demagógico/amorfa, conflictual/feudal, pero aclara que la simple caracterización de las instituciones sólo sobre la base de los criterios concernientes a origen y legitimidad del poder, no basta para los fines de estudiar sus formas reales de gerencia y orientación. Por otra parte encontramos los modelos de gestión universitaria dirigidos hacia las estrategias de desarrollo de las instituciones de educación superior, en los que predomina la dirección estratégica y los sistemas de soporte de la misma (Acosta, Becerra, y Jaramillo, 2017; Rabah, 2015; Almuiñas y Galarza, 2014; Choi, Lee y Yoo, 2010), así como los modelos de gestión operativa, donde se destaca la gestión por proyectos, que lidera la "Guía de los Fundamentos para la Dirección de Proyectos", (PMI, 2017) y los sistemas de gestión de la calidad. Todos estos modelos, estrategias y sistemas de gestión antes esbozados constituyen valiosas herramientas, que si se aplican consecuentemente en el ámbito de la educación superior, posibilitan el desarrollo universitario. Sin embargo las instituciones de educación superior requieren de un modelo conceptual que integre los elementos esenciales que posibilitan el desarrollo universitario armónico en su conjunto, a los efectos que sus autoridades puedan conducir acertadamente la dirección estratégica de la organización, sin desechar las virtudes que ofrecen el resto de los modelos y estrategias que se aplican en los diferentes procesos.

En este trabajo se presenta la aplicación de la Pirámide del Desarrollo Universitario en la Universidad de Otavalo, como modelo de gestión estratégica que integra las áreas de resultados clave, las restricciones de desarrollo, el contexto institucional y la dirección estratégica de la organización; modelo que se ha podido conceptualizar como resultado de una investigación previa desarrollada durante más de 15 años en la conducción exitosa de la dirección estratégica de las instituciones de educación superior siguientes: 1) en el Instituto Superior Politécnico José Antonio Echeverría de Cuba, la gestión universitaria realizada posibilitó que en el periodo del 1988-1999 el Instituto alcanzara y se mantuviera en el primer lugar del ranking de la educación superior cubana; 2) en el Ministerio de Educación Superior (MES) de Cuba, en su planificación estratégica a partir del período 2003-2007; 3) en la Universidad Agraria de la Habana en el 2004-2006, en que la dirección estratégica permitió en solo tres años, pasar del duodécimo al quinto lugar del ranking del MES; y 4) en la Universidad de las Ciencias Informáticas de Cuba en 2009-2011, recibiendo una Evaluación Institucional por el MES de satisfactoria en el 2010.

La hipótesis de la investigación es; "la aplicación del Modelo de la Pirámide de Desarrollo Universitario en la gestión estratégica Universidad de Otavalo contribuye a mejorar los resultados de la institución relacionados con el claustro, la formación académica, la investigación, la vinculación con la sociedad y el aseguramiento logístico". El objetivo general de la investigación es determinar en qué medida ocurre dicha contribución; como variable independiente se tiene por tanto la aplicación del modelo en la gestión estratégica de la Universidad de Otavalo y como variable dependiente el conjunto de los resultados de la institución en el transcurso del periodo de planificación.

\section{METODOLOGÍA}

El análisis documental de los resultados de las fases críticas, cíclicas y en espiral de la investigación previa realizada en las instituciones antes mencionadas, posibilitó identificar, mediante la utilización del método de análisis y síntesis, los principales elementos y su interrelación, que resultaban determinantes para la toma de decisiones en la gestión universitaria, lo que permitió definir a la Pirámide del Desarrollo Universitario como un modelo fundamental para la gestión estratégica de las instituciones de educación superior (Romillo y Polaino, 2018). Realizando una investigación correlacional con diseño cuasi experimental, partiendo de la hipótesis planteada, se aplicó el modelo de la Pirámide del Desarrollo Universitario en el plan estratégico de desarrollo institucional 2015-2020, siguiendo la metodología que se representa en el esquema de la figura 1 y que se explica a continuación. 


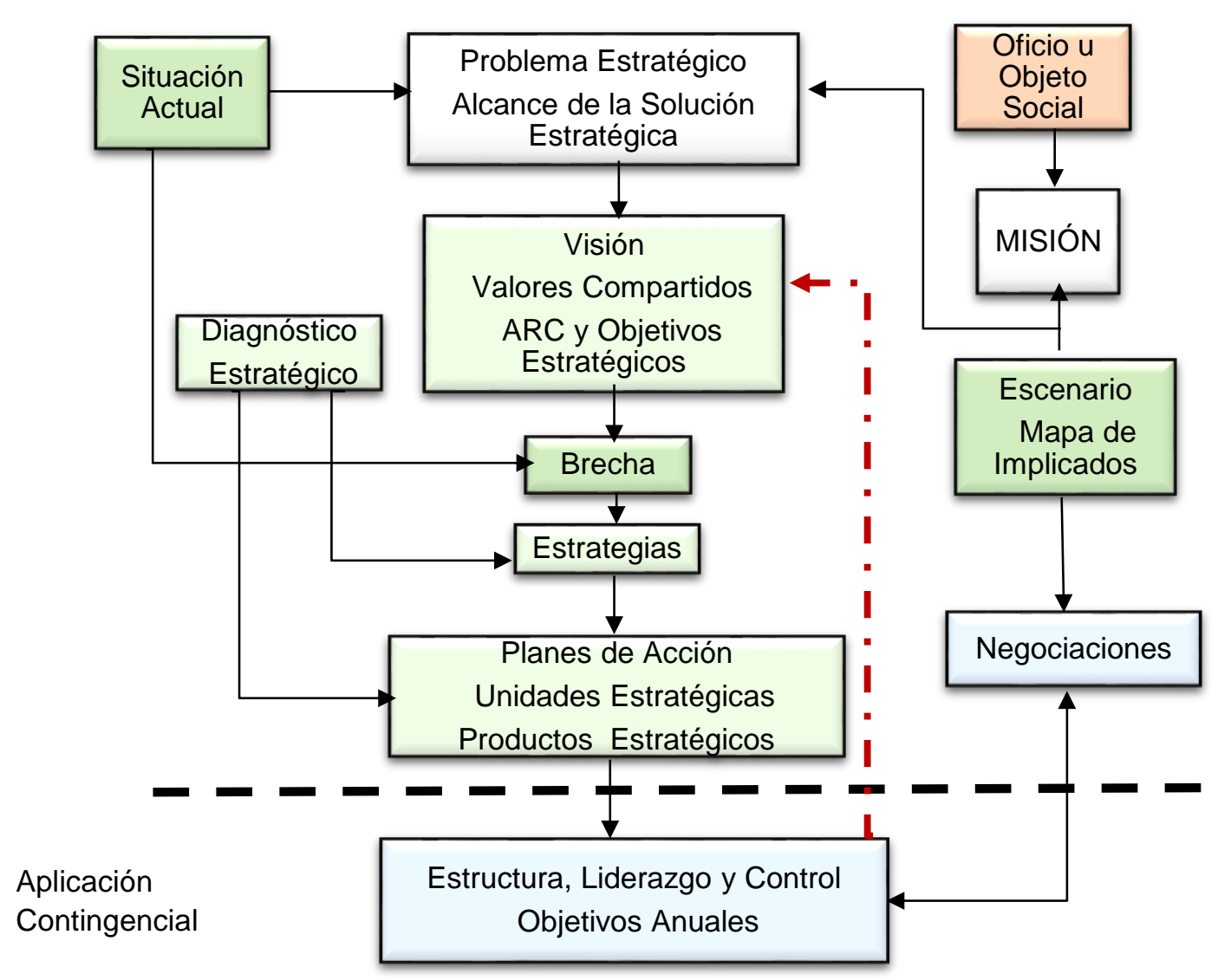

Fig. 1: Esquema para la Dirección Estratégica. Fuente: (Universidad de Otavalo, 2015)

A partir del objeto social se define la Misión, que constituye la razón de ser de la institución. Con ésta, analizando la situación actual que presenta la organización y el escenario más probable para el periodo de planificación, se identifica el problema estratégico y el alcance de la solución estratégica, lo que posibilita plantear la Visión, de la que se derivan los objetivos estratégicos por áreas de resultados clave (ARC), con sus respectivos criterios de medida y grados de consecución que deben alcanzarse en el transcurso del periodo de planificación. La determinación de las áreas de resultados clave es un momento muy importante y decisivo en el proceso de la planificación estratégica, pues es cuando se requiere descubrir con elevada certeza los factores críticos de éxito (FCE) de la organización. Los indicadores que resulten del análisis de los factores críticos de éxito y conformen los criterios de medida de los objetivos estratégicos de cada área de resultado clave, serán a su vez los que caractericen las entradas y salidas del Modelo de la Pirámide del Desarrollo Universitario, ya que el mismo forma parte de la Dirección Estratégica y por tanto se instrumenta a través del Plan Estratégico de Desarrollo Institucional (PEDI) y en los planes operativos anuales (POA).

La comparación de los valores de los indicadores que se deriven de los factores críticos de éxito, es lo que permite determinar el avance del desarrollo universitario en la medida que transcurre el periodo objeto de análisis. Los valores de dichos indicadores al inicio del periodo estarán dados por lo que en el gráfico se denomina situación actual y constituyen la entrada inicial de información al modelo. Los resultados que cada año se obtengan en cada indicador serán las salidas, que permiten evaluar el mencionado avance en la aplicación del modelo. Los indicadores de las áreas de resultados clave que caracterizan las entradas y salidas del modelo pueden variar de un periodo intermedio de planificación a otro, atendiendo a las oportunidades y amenazas que presenta el entorno y a las debilidades y fortalezas internas detectadas, pero sin renunciar al cumplimiento de la visión y de los objetivos estratégicos trazados. Los principales indicadores resultantes del análisis realizado en el caso de la Universidad de Otavalo fueron los siguientes: cinco pertenecientes al área de resultados clave del claustro de profesores; seis vinculados con el área de formación académica; tres relacionados con las investigaciones; dos en el área de vinculación con la sociedad y dos con el aseguramiento logístico; todos identificados en la tabla 1 de resultados de la aplicación del modelo.

El diagnóstico estratégico posibilita trazar las estrategias y los planes de acción que permiten vencer la brecha existente entre la situación actual y la proyectada en la visión, dando cumplimiento a los objetivos estratégicos. Para la realización del diagnóstico estratégico debe realizarse un levantamiento de la mayor información 
objetiva y confiable, a partir de la cual se debe realizar un análisis participativo que contemple el máximo posible de los involucrados, constituyendo una herramienta muy conveniente la Matriz FODA, que posibilita el cruce estratégico de la que se derivan las principales estrategias. En el caso de la Universidad de Otavalo la muestra de involucrados se calculó por la fórmula del muestreo probabilístico aleatorio simple para poblaciones finitas, con un nivel de confianza del $95 \%$, margen de error del $5 \%$ y variabilidad positiva del 0,5 , arrojando un total de 164 implicados, con la correspondiente composición de profesores, trabajadores y estudiantes.

En el proceso de planificación se precisan también los valores compartidos, unidades estratégicas y productos estratégicos, que la institución debe plantearse para los próximos años. Concluida la etapa de planificación comienza su aplicación atendiendo a las contingencias que surjan, a través de los planes operativos anuales ejecutados y controlados por toda la estructura, mediante un liderazgo transformacional de los jefes, en su interacción y negociaciones con todos los implicados. La validación de los resultados obtenidos en los diferentes indicadores de las áreas de resultados clave utilizados para valorar la contribución del Modelo de la Pirámide de Desarrollo Universitario en la gestión de la universidad, se realizó mediante los informes de cumplimento de los planes operativos anuales, aprobados por el consejo universitario de la institución.

Para la valoración integral del conjunto de los resultados de los indicadores de las áreas de resultados clave en la contribución del Modelo de la Pirámide de Desarrollo Universitario en la gestión de la institución, se utilizó la evaluación externa del Consejo de Evaluación, Acreditación y Aseguramiento de la Calidad de la Educación Superior (CEAACES) de Ecuador (CEAACES, 2016) cuya metodología analítica se sustenta en el análisis multicriterio (Figueira, Greco, y Ehrgott, 2005). Resultaba imprescindible vincular la contribución del Modelo a los resultados de la acreditación de la institución, nunca antes alcanzada.

\section{LA PIRÁMIDE DEL DESARROLLO UNIVERSITARIO}

La Pirámide del Desarrollo Universitario se conceptualiza como un modelo fundamental para la gestión estratégica de las instituciones de educación superior, que permite integrar, como se muestra en el esquema de la figura 2, cuatro componentes esenciales para el desarrollo universitario armónico, estos son: 1) las áreas de resultados clave, ubicadas en el interior de la pirámide; 2 ) las restricciones de desarrollo, conformando los lados; 3) el contexto institucional; y 4) la dirección estratégica de la organización, que enmarca y abarca a los anteriores elementos. A continuación se explican cada uno de los componentes de la Pirámide de Desarrollo Universitario, que posibilitan en su conjunto guiar la gestión de la institución de educación superior y sus procesos hacia planos superiores deseados del desarrollo universitario.

\section{Áreas de Resultados Clave}

Integradas por el claustro de profesores, los procesos de las funciones sustantivas universitarias (formación, investigación y vinculación con la sociedad) y el aseguramiento logístico, son donde se encuentran los factores críticos de éxito y en las que se deben concentrar los principales objetivos de trabajo, que posibiliten mover a la organización en la dirección estratégica correcta. No deben ser muchas para definir bien la dirección principal, deben actuar sobre el $20 \%$ de los elementos que producen el $80 \%$ de los resultados del cambio requerido, según el Principio de Pareto o regla de 80/20 (Delors, 2016) y pueden ser diversas en dependencia de: la situación que presente la institución y sus procesos, el escenario de desenvolvimiento y los cambios para alcanzar el estado de desarrollo deseado; sin embargo, como resultado de la investigación previa realizada en las cuatro instituciones mencionadas en la introducción, se ha identificado que en el caso de una institución de educación superior, las que imprescindiblemente tienen que tenerse en cuenta son las que se presentan en el interior de la pirámide de la figura 2 y se describen a continuación.

Claustro de profesores, que ocupa la cúspide de la pirámide del desarrollo universitario, pues al ser la educación superior la razón de ser de la institución, el profesor resulta el factor más importante para el desarrollo del proceso de enseñanza aprendizaje y la realización exitosa de los diferentes procesos sustantivos universitarios. La universidad contemporánea requiere de un profesor con alto nivel científico, y elevada maestría pedagógica, que sepa conducir e integrar los procesos sustantivos y los de apoyo, en aras de lograr los resultados esperados.

El profesor universitario es más que un docente, como a veces se le suele denominar, debe ejercer la labor educativa con calidad, destacarse en la investigación científica, promover la vinculación con la sociedad en función del desarrollo sostenible y a su vez, debe ser capaz de gestionar la logística e integración de los procesos universitarios que atiende. Su papel ya no es únicamente dar buenas clases y enseñar con calidad su disciplina a los estudiantes, en la actualidad es el principal gestor de los procesos de formación, 
investigación y de vinculación con la sociedad o extensión universitaria de la institución de educación superior.

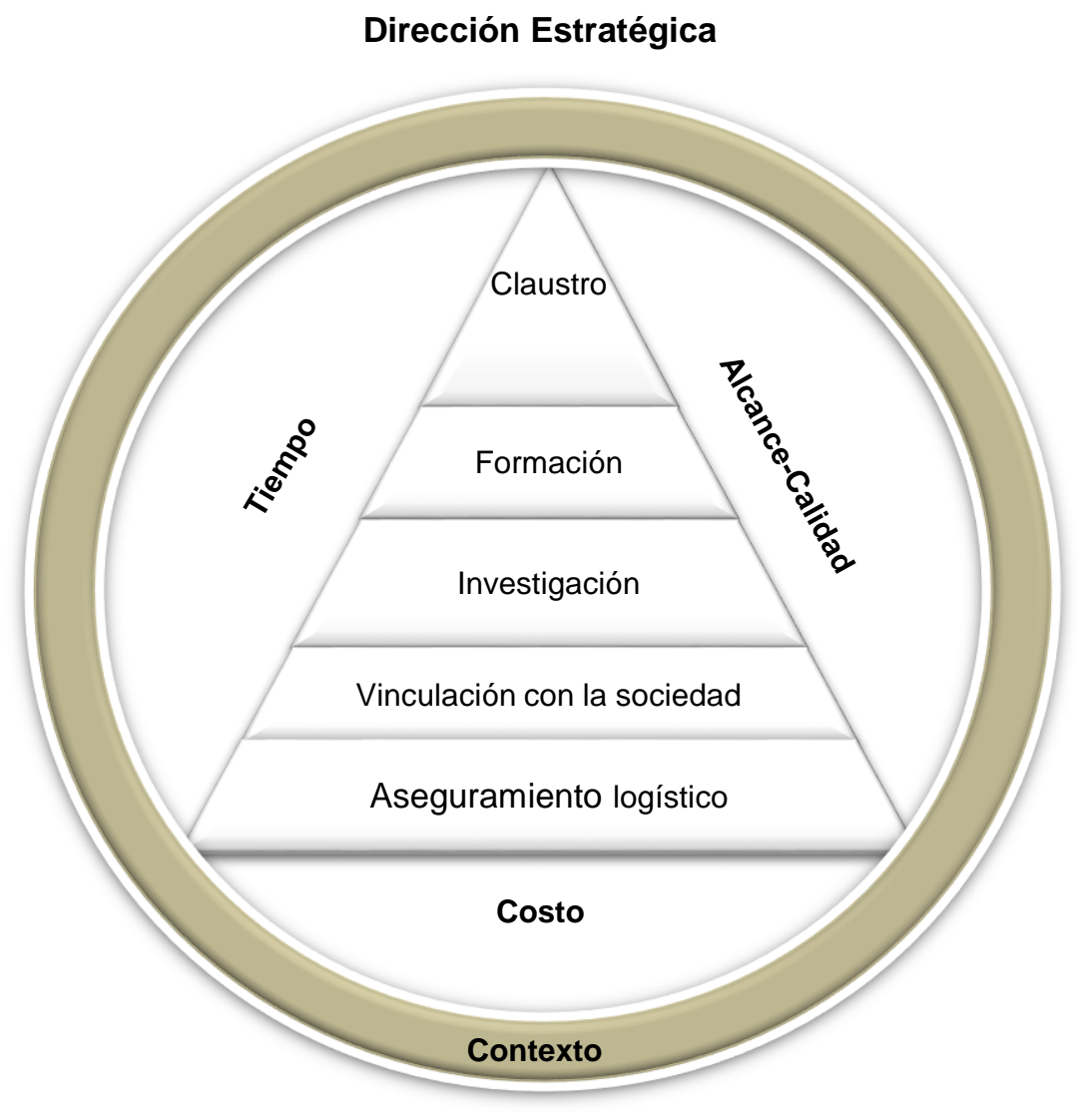

Fig. 2: Pirámide de Desarrollo Universitario. Fuente: (Romillo y Polaino, 2018)

Es generalizado el reconocimiento que lo más importante que tienen las organizaciones es su talento humano, lo que adquiere mayor relevancia en el caso de los profesores de las instituciones de educación superior, por desenvolverse en el ámbito de la gestión del conocimiento. Se requiere por tanto gestionar eficientemente la adecuada composición, preparación, actuación y evaluación del claustro de profesores, como principal recurso y gestor que posee la universidad, convirtiéndose así en la principal área de resultados clave que debe tenerse en cuenta en el modelo de gestión de la universidad y en la que deben proponerse criterios de medida concretos y retadores, en cuanto porcentaje de doctores en ciencias en el claustro, profesores en formación hacia el cuarto nivel, superación pedagógica, producción científica de los profesores, tránsito hacia categorías y niveles académicos superiores, y otros que posibiliten la mejora permanente del claustro de profesores.

Los procesos correspondientes a las funciones sustantivas universitarias, que siguen en orden de importancia en el interior de la pirámide de desarrollo universitario: formación integral y continua de profesionales, investigación científica y vinculación con la sociedad; cada uno de estos procesos por si solo y a su vez de forma integrada, debe gestionarse como área de resultados clave.

La universidad tiene sus antecedentes en las instituciones educativas del Mundo Antiguo y surge en Europa en la época medieval, a principios del Siglo XII en las Universidades de Bolonia (1119), Paris (1150) y Oxford (1167), como asociaciones libres de hombres de diferentes lugares que se proponían cultivar "aprender los saberes". La estrecha relación entre la docencia y la investigación, surge del modelo de Guillermo Humboldt en la Universidad de Berlín (1810) y en el Siglo XIX surge la tercera misión de la universidad, que varios autores (Loi y Di Guardo, 2015; Görason, Maharajh y Schmoch, 2009), valoran como el fortalecimiento de la función social de la universidad y su contribución al desarrollo de la sociedad.

Aunque en los países en desarrollo aún se pueden encontrar universidades que sólo se dedican a la formación de profesionales, con escaso vínculo de la docencia con la investigación y con la vinculación con la sociedad, en la actualidad no es concebible una universidad que se respete por su calidad, que no realice e integre las tres funciones sustantivas universitarias, lo que es avalado a través de los procesos de acreditación nacionales e internacionales. Los procesos de formación, investigación y vinculación con la sociedad, 
convertidos en áreas de resultados clave, deben contar con objetivos, metas, criterios de medida, grados de consecución y planes de acción concretos que posibiliten la mejora continua y resultados cada vez superiores. Aunque cada uno de estos procesos sustantivos se trate como área de resultado clave independiente, no debe desatenderse su integración e interdependencia, pues en su unidad está la fuerza del desarrollo de la institución de la educación superior.

Aseguramiento Logístico, aparece en la base de la pirámide del desarrollo universitario, como el sustento requerido para desarrollar al claustro de profesores y para llevar a cabo con calidad los procesos sustantivos universitarios. Entre los principales aseguramientos que deben ser atendidos están los recursos humamos y el clima laboral; la organización del trabajo; la base material en cuanto aulas, laboratorios, bibliografía y otros recursos materiales; el bienestar y ambiente universitario; la tecnología disponible; el aseguramiento y diversificación de las fuentes financieras; la comunicación interna y externa; el respaldo legal; y la gestión de riesgos, entre otros.

Aunque el claustro de profesores es el factor más importante para lograr buenos resultados en las funciones sustantivas universitarias, el avance de la universidad depende también en gran medida del nivel de aseguramiento logístico, y como área de resultado clave también debe contener las metas y acciones fundamentales que posibiliten el mayor desarrollo de la institución y sus procesos. A los efectos del presente trabajo se seleccionó como indicador integrador de esta área el incremento del presupuesto económico destinado al aseguramiento logístico del plan operativo anual, así como las asignaturas con textos básicos y complementarios actualizados en la biblioteca, por su incidencia en la formación académica.

Las cinco áreas de resultados clave antes explicadas fueron contempladas como parte de la aplicación del modelo en el plan estratégico de desarrollo institucional de la Universidad de Otavalo, correspondiente al periodo 2015-2020.

\section{Restricciones de desarrollo}

Universalmente aceptadas en la gestión de proyectos, conformando el denominado Triángulo del Proyecto (Microsoft, 2016 y Lledó, 2013) son válidas y están presentes en la gestión de la universidad y sus procesos. La estrecha interrelación entre alcance, calidad, tiempo y costo, está presente en la gestión de los procesos para alcanzar cualquier producto, meta o resultado de las diferentes áreas de resultados clave, tanto a nivel estratégico a largo o mediano plazo, como operativo a corto plazo, lo que se sustenta en que se cumplen las características distintivas de naturaleza única y temporalidad, que promulga la teoría de la gestión de proyectos (PMI, 2017).

Limitaciones financieras, pueden condicionar el alcance y la calidad de algún resultado que se pretenda obtener, así como extender el tiempo de duración para lograrlo. De igual forma resultados de gran alcance y calidad, por lo general implican elevados costos y plazos de tiempo prolongados, así como incumplimientos en los tiempos establecidos para lograr un resultado de calidad originan mayores costos en la ejecución.

Por lo anteriormente expresado, en la gestión universitaria para la obtención de los resultados de las diferentes áreas de resultados clave, no se deben obviar las restricciones para el desarrollo que implican alcance, calidad, tiempo y costo, deben planificarse y recibir el debido seguimiento y control. En la figura 2 se encuentran conformando los lados de la pirámide que contiene a las áreas de resultados clave y la forma triangular condiciona que la variación de alguna de estas restricciones, implicará necesariamente cambio en al menos otra de ellas; así por ejemplo, la actualización del aseguramiento bibliográfico en el tiempo estará condicionado por la oferta académica, la cantidad de estudiantes y la disponibilidad financiera, evidenciándose con ello la interrelación entre estas restricciones.

En el caso que nos ocupa de la Universidad de Otavalo, dichas restricciones de desarrollo se ven reflejadas en la interrelación del alcance y la calidad de las metas de los diferentes indicadores planteados, con los presupuestos para llevarlos a cabo, a lo largo del periodo de planificación.

\section{Contexto institucional}

La vinculación con la sociedad adquiere mayor importancia en la gestión de la universidad contemporánea, pues en la actualidad el servicio que se presta no es únicamente el de formación que se le brinda a los estudiantes y los profesionales que egresan, resulta necesario atender además: las necesidades de superación continua y educación de posgrado de los profesionales; las investigaciones, generación de conocimientos y producción científica, intrínsecamente ligadas a la calidad de los procesos formativos de grado y posgrado; a través de la vinculación con la sociedad se requiere poner el alto potencial académico y científico de la universidad al servicio del desarrollo sostenible de la nación, la región y la localidad, mediante 
consultorías y asesorías científico-técnicas; actividades culturales, deportivas y sociales de extensión universitaria también impactan en el entorno; la participación en redes y las relaciones locales nacionales e internacionales forman también, junto a otras actividades, el complejo entramado que conforma el contexto en el que está inmersa la institución de educación superior.

En las circunstancias mencionadas, resulta muy amplía la definición del mapa de involucrados tanto externos como internos, así como muy complejo lograr la satisfacción de los mismos con los servicios prestados por la universidad. Resulta importante no solo brindar un producto o servicio de calidad, sino además lograr la satisfacción de los clientes, que muchas veces no se logra por una ineficaz gestión de interesados, aunque se cuente con un buen resultado. La identificación de los involucrados, internos o externos, partidarios u opositores, sus necesidades e intereses, su grado de influencia, la comunicación con los mismos y el establecimiento de estrategias de abordaje de ganar-ganar, son elementos importantes a considerar en la gestión de interesados que conforman el contexto de la institución de la educación superior.

En el caso particular de la Universidad de Otavalo, que se encuentra enclavada en un territorio donde predomina la población indígena, el contexto institucional se ha tenido muy en cuenta y en especial la interculturalidad, formando parte incluso de la misión, visión, principios fundacionales y los objetivos estratégicos, contenidos en sus Estatutos y en el Modelo Educativo de la institución.

\section{Dirección estratégica}

La operatividad diaria del trabajo es inevitable y necesaria realizarla con agilidad y efectividad, pues cada día surgen problemas internos, externos e indicaciones de los niveles superiores que deben atenderse para que la organización funcione con la calidad e imagen requeridas. Sin embargo las actividades operativas no deben opacar a la dirección estratégica y los líderes, con sus respectivos consejos de dirección, están obligados a instrumentar el seguimiento a los planes estratégicos. En la actualidad las organizaciones están sometidas a condiciones turbulentas en entornos muy cambiantes, por lo que la planificación estratégica no debe aplicarse esquemáticamente con la formalidad, racionalidad y estructuración como se aplicaba la planificación tradicional, se requiere en la actualidad una aplicación tipo contingencial, que sin perder la visión estratégica, sea suficientemente flexible y transformacional que aproveche todas las oportunidades que ofrece el entorno y neutralice las amenazas, con vista a lograr los objetivos estratégicos de desarrollo que se ha trazado la organización. Son requisitos indispensables para ello el pensamiento estratégico y el liderazgo transformacional. (Aznar, 2017; Osson y Simerson, 2015).

Existen muchos criterios e incluso escuelas sobre la Dirección Estratégica. Mintzberg y otros (1999), ofrecen una síntesis muy completa analizando en 9 dimensiones, 10 escuelas que agrupa en tres posiciones: prescriptivas, descriptivas e integral. En cuanto a la implementación y control de las estrategias organizacionales también son muchos los enfoques y herramientas, destacándose la orientación de la dirección estratégica hacia la mejora continua de la calidad de los procesos universitarios (Almuiñas y Galarza, 2014) y el Cuadro de Mando Integral desarrollado por Kaplan y Norton (2007), aplicado por otros autores (Comas, Nogueira y Medina, 2014).

La planificación estratégica no puede concluir simplemente con un documento bien elaborado que se engavete y se desempolve cuando los órganos de control lo soliciten. Debe ser una guía de acción para la gestión de la institución de educación superior durante todo el periodo para el cual se proyectó el plan. Tampoco su aplicación debe ser rígida y dogmática, debe tener en cuenta las contingencias que cotidianamente surgen, que pudieran poner en peligro el cumplimiento de la visión y de los objetivos estratégicos. La denominada aplicación contingencial debe centrarse fundamentalmente en la permanente búsqueda de estrategias y acciones que posibiliten alcanzar las metas trazadas, contrarrestando los inconvenientes que cotidianamente surgen, o aprovechando las oportunidades que se presenten. Renunciar a metas que pongan en riesgo a los objetivos estratégicos y la visión, debido a inconvenientes que surgen, debe ser siempre la última opción; antes deben encontrarse las vías para solucionar el problema que afecta el cumplimiento de la meta.

\section{RESULTADOS DE LA APLICACíón DEL MODELO DE GESTIÓN PIRAMIDE DE DESARROLLO UNIVERSITARIO EN LA UNIVERSIDAD DE OTAVALO}

Utilizando la metodología antes explicada, se aplicó el modelo de la Pirámide de Desarrollo Universitario en la dirección estratégica de la Universidad de Otavalo, a través de su plan estratégico de desarrollo institucional del 2015-2020 y los planes operativos anuales, arrojando los resultados que se analizan a continuación, cuya fuente de información fueron los informes de cumplimiento de los planes operativos anuales correspondientes a los años 2015, 2016 y 2017 a través del Sistema de Información Estratégico de Gestión Universitaria (SIEGU) de la propia universidad (Universidad de Otavalo, 2017). 
En la tabla 1 se puede apreciar el comportamiento de los indicadores seleccionados para determinar la contribución del modelo a los resultados de la institución, durante los tres años que se evaluó. Los indicadores relacionados con la composición y preparación del claustro de profesores han mejorado sistemáticamente durante los tres años que se está aplicando este modelo de gestión. Con respecto al año 2014, el porcentaje de profesores de tiempo completo en el claustro se incrementó en 9\%; en cuanto a los profesores de tiempo completo y parcial con títulos de cuarto nivel ascendió el porcentaje en un $21 \%$ y la presencia de PhD entre los profesores de tiempo completo exhibe un crecimiento del $18.1 \%$.

Tabla 1: Resultados de la aplicación del Modelo de la Pirámide del Desarrollo Universitario en la Universidad de Otavalo. Los resultados son expresados en porcentaje

\begin{tabular}{|c|c|c|c|c|}
\hline \multirow{2}{*}{ Indicador } & \multicolumn{4}{|c|}{ Año } \\
\hline & 2014 & 2015 & 2016 & 2017 \\
\hline Profesores de tiempo completo en el claustro & 63 & 70,1 & 71 & 72 \\
\hline Profesores de tiempo completo y parcial con títulos de cuarto nivel & 60 & 72,9 & 73 & 81 \\
\hline Profesores de tiempo completo con grado de $\mathrm{PhD}$ & 5 & 19,2 & 20 & 23,1 \\
\hline Implementación del plan de trabajo anual del profesor & - & 90 & 90 & 100 \\
\hline Profesores del claustro evaluados satisfactoriamente & - & 96 & 97 & 98 \\
\hline Carreras rediseñadas & - & 42 & 57,1 & 100 \\
\hline Eficiencia académica & 27 & 29 & 31 & 50 \\
\hline Incremento de estudiantes matriculados vs año 2014 & - & 16.6 & 42.3 & 99 \\
\hline Cumplimiento del nuevo sistema de trabajo docente metodológico & - & 50 & 80 & 100 \\
\hline Satisfacción de los estudiantes con la calidad de la formación recibida & 78 & 85.8 & 91.6 & 93.46 \\
\hline Profesores vinculados a las investigaciones & 30 & 53 & 57 & 74 \\
\hline Incremento de las publicaciones de los profesores vs año 2014 & - & 25 & 62.5 & 412.5 \\
\hline Estudiantes vinculados a las investigaciones & 30 & 30 & 35 & 40 \\
\hline Incremento de los proyectos de vinculación vs año 2014 & - & 42.8 & 157.1 & 171.4 \\
\hline Incremento de estudiantes en proyectos de vinculación vs año 2014 & - & 49.5 & 158.8 & 175.7 \\
\hline Asignaturas con textos básicos y complementarios actualizados en la biblioteca & 40 & 40 & 75 & 85 \\
\hline $\begin{array}{l}\text { Incremento del presupuesto económico de aseguramiento del plan operativo anual } \\
\text { con respecto al año } 2014\end{array}$ & - & 16.45 & 21.72 & 27.41 \\
\hline
\end{tabular}

Se instrumentó el plan de trabajo del profesor, integrando al mismo su superación y preparación, lo que en el año 2014 no existía, así como la evaluación del desempeño de los docentes, apreciándose resultados favorables en el periodo, sobre todo en cuanto a la evaluación de los profesores que ha llegado a alcanzar el $98 \%$ con evaluación satisfactoria. Todas estas mejoras logradas en la composición y preparación del claustro de profesores, han sido decisivas en los resultados también favorables alcanzados en los procesos sustantivos de la universidad.

Como se aprecia en la tabla 1, los indicadores relacionados con la formación de los profesionales han ido creciendo durante los tres años en que se está aplicando este modelo de gestión: todas las carreras fueron rediseñadas, incremento en $23 \%$ de la eficiencia académica, cumplimiento del nuevo sistema de trabajo docente metodológico, incremento en $99 \%$ de la matrícula con respecto al año 2014 y sobre todo el indicador que resulta más integrador, la satisfacción de los estudiantes en relación a la calidad de la formación que están recibiendo, medida a través del SIE-GU con encuestas anónimas a la totalidad de los estudiantes; indicador que ha ido creciendo hasta alcanzar el umbral de la excelencia con un 93, $46 \%$. Aunque no se debe absolutizar este indicador y se realizan controles a clase y encuentros con estudiantes, para completar la información, resulta evidente que se ha avanzado en la calidad de la formación académica. (Universidad de Otavalo, 2017)

En los procesos sustantivos de la investigación y la vinculación con la sociedad también se aprecian avances en la tabla 1: el porcentaje de profesores y estudiantes vinculados a las investigaciones se ha incrementado en un 44 y $10 \%$ respectivamente; crecieron también en $412,5 \%$, las publicaciones de los profesores con respecto al año 2014, incluyendo las de base de datos internacionales, así como, se incrementó con respecto a dicho año en un $175,7 \%$ la cantidad estudiantes incorporados a proyectos de vinculación con la sociedad, 
todo lo cual se legró a través de las estrategias y planes de acción planteadas para estas dos áreas de resultados clave (Universidad de Otavalo, 2017).

En cuanto al aseguramiento logístico destacan el incremento de un 45\% de las asignaturas con textos básicos y complementarios actualizados en la biblioteca y el incremento en un $27.41 \%$ del presupuesto económico destinado al aseguramiento logístico del plan operativo anual con respecto al año 2014, como se aprecia en la tabla 1. Todos estos resultados, integrados en la metodología multicriterio aplicada en la evaluación externa por el Consejo de Evaluación, Acreditación y Aseguramiento de la Calidad de la Educación Superior de Ecuador, contribuyeron al logro más importante, que fue la obtención de la condición de Universidad Acreditada, reconocida en octubre del 2016, marcando un antes y un después, a partir de la cual la institución se ha trazado una nueva proyección hacia niveles superiores de calidad de desarrollo universitario

Se comprueba por tanto la hipótesis planteada, pues la aplicación del Modelo de la Pirámide de Desarrollo Universitario en la gestión estratégica Universidad de Otavalo contribuyó, a mejorar los resultados de la institución en las áreas de claustro, formación académica, investigación, vinculación con la sociedad, aseguramiento logístico y en su conjunto posibilitó obtener la condición de universidad acreditada, nunca antes alcanzada por la institución.

\section{CONCLUSIONES}

De acuerdo a los resultados de la investigación, de su discusión y análisis, se pueden extraer las conclusiones siguientes:

1) se ha conceptualizado la Pirámide del Desarrollo Universitario como un modelo fundamental de gestión estratégica que integra las áreas de resultados clave, las restricciones de desarrollo, el contexto institucional y la dirección estratégica de las instituciones de educación superior;

2) utilizando la metodología de investigación explicada, se aplicó el modelo de la Pirámide de Desarrollo Universitario en la dirección estratégica de la Universidad de Otavalo, a través de su plan estratégico de desarrollo institucional del 2015-2020 y los planes operativos anuales;

3) la aplicación durante tres años del Modelo de la Pirámide de Desarrollo Universitario en la gestión estratégica Universidad de Otavalo contribuyó a mejorar los resultados de la institución en las áreas de claustro, formación académica, investigación, vinculación con la sociedad, aseguramiento logístico y en su conjunto posibilitó obtener la condición de universidad acreditada, nunca antes alcanzada por la institución, con lo que se comprobó la hipótesis planteada en la investigación

\section{REFERENCIAS}

Abreu O., M.E. Naranjo, B.S. Rhea y M. C. Gallegos, Modelo Didáctico para la Facultad de Ciencias Administrativas y Económicas de la Universidad Técnica del Norte en Ecuador, doi: 10.4067/S0718-50062016000400002, Revista Formación Universitaria, 9(4), 3-10 (2016)

Acosta, L.A., F. Becerra y D. Jaramillo, Sistema de Información Estratégica para la Gestión Universitaria en la Universidad de Otavalo (Ecuador), doi: 10.4067/S0718-50062017000200011, Revista Formación Universitaria, 10(2), 103-112 (2017)

Acosta, L.A., O. Abreu y M. Coronel, Sistema de Formación Pedagógica en la Universidad de Otavalo en Ecuador, doi: 10.4067/S0718-50062015000200007, Revista Formación Universitaria, 8 (2), 43-52 (2015)

Almuiñas, J.L. y J. Galarza, Dirección Estratégica y Calidad en la Educación Superior (potencialidades para su desarrollo,

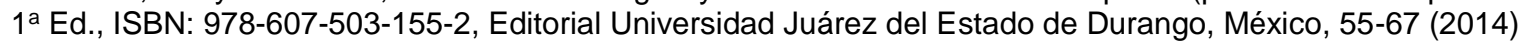

Aznar, F.M., Repensando el liderazgo estratégico, El liderazgo estratégico como liderazgo transformacional -4a-, Documento de Análisis 40/2017. ieee.es, (2017)

CEAACES, Informe final del proceso de evaluación, acreditación y re categorización 2016 de la U. de Otavalo (2016)

Choi, S.Y., H. Lee y Y. Yoo, The Impact of Information Technology and Transitive Memory Systems on Knowledge Sharing, Application, and Team Performance: A Field Study, MISQuarterly, 34(4), 855-870 (2010)

Comas, R., D. Nogueira y A. Medina, El control de gestión y los sistemas de información: propuesta de herramientas de apoyo, Ingeniería Industrial, 35(2), 214-228 (2014)

Dan, M.C., The Third Mission of Universities in the Development Strategy of Vienna City, Rev. Informática Económica, ISSN: 1453-1305, 16(4), 49-56 (2012)

Delors, A. e I. Van Steenkiste, El principio de Pareto: Optimice su negocio con la regla del 80/20. eBook Mobipocket, Editorial 50Minutos.es (2016) 
Etzkowitz, H., The entrepreneurial university wave: from ivory tower to Global Economic Engine, doi: 10.5367/ihe.2014.0211, Journal of Industry and Higher Education, 28 (4), 223-232 (2014)

Figueira, J., S. Greco y M. Ehrgott, Multiple criteria decision analysis: state of the art surveys, volume 78. Springer (2005)

Görason, B., R. Maharajh y U. Schmoch, New activities of universities in transfer and extension: multiple requirements and manifold solutions. doi.org/10.3152/030234209X406863, Journal Science and Public Policy, 36(2), 157-164 (2009)

Henningsen, B., J. Schlaeger, y H-E. Tenorth, Humboldts Model: The Future of the Universities in the World of Research: Conference Report, $1^{a}$ Ed., 201-231, BWV Verlag, Berlin, Germany (2013)

Kaplan, R.S. y D. P. Norton, Using the Balanced Scorecard as Strategic Management System, Boston: Best of Harvard Business Review, 1-15, July-August (2007)

Larrea, E., Modelo de organización del conocimiento por dominios científicos, tecnológicos y humanísticos (en línea), Consejo de Educación Superior, Ecuador, (2014)

Lledó, P., Restricción Triple, Cuádruple, Quíntuple... de un proyecto (2013)

Loi, M. y M. Di Guardo, The third mission of universities: An investigation of the espoused values. (SPP), Science Public Policy, 42(6) (2015)

Lolas, F., Sobre los modelos de gestión universitaria. ISSN-e 0717-4004, Revista Calidad en la Educación, (24) 37-45, Chile (2006)

Microsoft., El triángulo del proyecto. Software de administración de proyectos Ms Project (2016)

Mintz S., Four Emergent Higher Education Models; Extension, distributed, the cloud and student-centered, (on line) Inside Higher ED Company, Washington DC, USA (2014)

Mintzberg, H., B. Ahlstrand y J. Lampel, Safari a la estrategia. Una visita guiada por la jungla del management estratégico, 4-9, Ediciones Granica, Argentina (1999/2003)

Montesinos, P., J.M. Carot, J.M. Martínez y F. Mora, Third Mission Ranking for World Class Universities: Beyond Teaching and Research, doi: 10.1080/03797720802254072, Journal of Higher Education in Europe, 33(2-3), $259-271$ (2008)

Nowotny, H., P. Scott y M. Gibbons, Mode 2.The New Production of Knowledge, Minerva, ISSN: 0026-4695, 4(41), 179194 (2003)

Osei-Bryson, K-M., G. Mansingh, y L. Rao, Understanding and Applying Knowledge Management and Knowledge Management Systems in Developing Countries: Some Conceptual Foundations in Knowledge Management for Development, doi: 10.1007/978-1-4899-7392-4_1, Springer Science + Business Media, 35,1-15 (2014)

Osson A.K. y B.K. Simerson, Leading with Strategic Thinking: Four Ways Effective Leaders Gain Insight, Drive Change, and Get Results. Wiley, (2015)

Patel, V.K. y A.B. Gorvadiya, Knowledge management: the need of modern organizations, Journal of Knowledge Management, 6(3), 259-261 (2014)

PMI, A Guide to the Project Management Body of Knowledge (PMBOK® Guide)-6ta Edition, Project Management Institute Inc., USA (2017)

Polaino, C. y A. Romillo, Vinculación con la Sociedad en la Universidad de Otavalo, Ecuador, doi.org/10.4067/S071850062017000300004, Revista Formación Universitaria, 10(3), (2017)

Rabah, I., Introduction of Managerialism into University Administration: Erosion of the Collegial Model,Shared Governance, and Academic Tenure. Global Journal of Management and Business Research: A Administration and Management, ISSN 0975-5853 (Print), ISSN: 2249-2249-4588 (Online) 15(12) Version 1, 0, USA (2015)

Romillo, A. y C. Polaino, La Pirámide de Desarrollo Universitario. XIV Taller Internacional "La educación superior y sus perspectivas". 11no. Congreso Internacional de Educación Superior Universidad 2018, Ministerio de Educación Superior, (810-819), La Habana, Cuba, 12-16 de febrero (2018)

Universidad de Otavalo, Informe de cumplimiento del Plan Operativo Anual (POA) de la Universidad de Otavalo, Ecuador. Año 2014, 2015, 2106 y 2017 (2017)

Universidad de Otavalo, Plan Estratégico de Desarrollo Institucional (PEDI) de la Universidad de Otavalo, período 20152020 (2015) 